\title{
Automatic segmentation of brain tissue based on improved fuzzy c means clustering algorithm
}

\author{
Zhuang Miao", Xiaomei Lin², Chengcheng Liu ${ }^{2}$ \\ ${ }^{1}$ Department of neurosurgery, China-Japan Union Hospital, Jilin University, Jilin, China; \\ ${ }^{2}$ Institute of electrical and electronic engineering, Changchun University of Technology, Changchun, China \\ Email: miaozhuang99@163.com, linxiaomei@mail.ccut.edu.cn, elaine2008study@sina.com
}

Received 9 November 2010; 12 November 2010; 23 November 2010.

\begin{abstract}
In medical images, exist often a lot of noise; the noise will seriously affect the accuracy of the segmentation results. The traditional standard fuzzy c-means(FCM) algorithm in image segmentation do not taken into account the relationship the adjacent pixels, which leads to the standard fuzzy c-means(FCM) algorithm is very sensitive to noise in the image. Proposed improved fuzzy c-means(FCM) algorithm, taking both the local and non-local information into the standard fuzzy c-means(FCM) clustering algorithm. The experiment results can show that the improved algorithm can achieve better effect than other methods of brain tissue segmentation.
\end{abstract}

Keywords: Local Information; Non-local Mean; Brain Tissue Segmentation

\section{INTRODUCTION}

Image segmentation is the key technology in image processing and analysis .In the medical field, with the imaging technology development and medical imaging application success in the clinical, image segmentation is playing an increasingly larger role. On the MRI (magnetic resonance imaging) brain images in the white matter (WM), brain gray matter (GM) and cerebrospinal fluid (CSF) such as the organizational structure of the correct segmentation in medical applications is of great significance. However, there is a lot of noise; the noise will seriously affect the accuracy of the segmentation results. The traditional standard FCM algorithm in image segmentation do not taken into account the relationship adjacent pixels, which leads to the standard FCM algorithm is very sensitive to noise in the image. Although some noise in the image can be removed in split before use of smoothing filters and other methods of, but in most cases, this approach is unwise, because some of the details in the image and edge information and noise may also be removed together by some standard filters. In order to reduce image noise affect to image and better for the partition of the segmented image, here we propose a new algorithm in this chapter, in the proposed new algorithm, take both the local and non-local information into the standard FCM clustering algorithm.

Non-local means algorithm (NL Means), who by Buades and other peoples as image denoising algorithm first proposed [1,2]. This algorithm attempts to use the image height of the redundant information to complete the work of digital image denoising, in other words, for each pixel in the image, we can find a lot of images with which had the same structure of adjacent domains samples, then we are dealing with these redundant pixels to be weighted average of the sample. Experimental results show that non-local means algorithm can successfully remove the image noise at the same time save the image in the more complete boundary information. However, in medical images, the boundary between different organizations is often blurred, and the details of the image, redundant information is not always present. In order to protect the image of the fine structure and details of the information and made them not be destroyed, in the use of non-local means algorithm at the same time, local information should also be considered.

\section{AUTOMATIC SEGMENTATION ASED ON IMPROVED FUZZY C MEANS CLUSTERING ALGORITHM}

\subsection{Improved Fuzzy C Means Clustering Algorithm Distance Function}

Through the standard FCM algorithm, we can see the final result is determined by the value of the data of the degree of membership, and degree of membership of data is determined by the distance function .Therefore, we can make conclusions, and the key in the FCM is the distance that data and cluster center. The proposed algo- 
rithm in this chapter, we will distance function in the standard FCM rewritten as

$$
D^{2}\left(x_{j}, v_{i}\right)=\left(1-\lambda_{j}\right) d_{l}^{2}\left(x_{j}, v_{i}\right)+\lambda_{j} d_{n l}^{2}\left(x_{j}, v_{i}\right)
$$

Among them, $d_{l}$ is the distance by the impact of local information, $d_{n l}$ has been affected by the distancenon-local information, $\lambda_{j}$ is used to control the proportion parameters between these two diatances, the valur range is $[0,1]$. For a pixel $x_{i}, N_{i}$ is selected with fixed-size local neighborhood structure, if the $x_{k}$ in the $N_{i}$ is very close to a pixel gray value of, the center pixel then $x_{i}$ should be affected largely by it, Otherwise, its impact should be very small to $x_{i}$. According to the above description, the distance formula affected by the local neighborhood information is:

$$
d_{l}^{2}\left(x_{j}, v_{i}\right)=\frac{\sum_{x_{k} \in N_{j}} w_{l}\left(x_{k}, x_{j}\right) d^{2}\left(x_{k}, v_{i}\right)}{\sum_{x_{k} \in N_{j}} w_{l}\left(x_{k}, x_{j}\right)}
$$

Where, $w_{l}\left(x_{k}, x_{j}\right)$ is the weightin in the neighborhood of each pixel,defined as:

$$
w_{l}\left(x_{k}, x_{j}\right)=e^{-\frac{\left|x_{k}-x_{j}\right|^{2}}{\delta^{2}}}
$$

Formula (3) is the variance of pixel gray value in the neighborhood $N_{i}$, use it to control the bending degree of curve.

All the distance dnl affected by the non-local information is the weighted average in all pixel input image I, calculated as:

$$
d_{n l}^{2}\left(x_{j}, v_{i}\right)=\sum_{x_{k} \in l} w_{n l}\left(x_{k}, x_{j}\right) d^{2}\left(x_{k}, v_{i}\right)
$$

Among them, the weighted values $w_{n l}\left(x_{k}, x_{j}\right)$ is determined by $x_{j}, x_{k}$ the similarity of pixels to $\sigma^{2}$. Generally speaking, $w_{n l}, w_{n l}\left(x_{k}, x_{j}\right)$ satisfy the following conditions:

$$
\begin{gathered}
0 \leq w_{n l}\left(x_{k}, x_{j}\right) \leq 1, \\
\sum_{x_{k} \in l} w_{n l}\left(x_{k}, x_{j}\right)
\end{gathered}
$$

\subsection{Improved Fuzzy C Means Clustering Algorithm Weight}

The similarity of Pixel $x_{k}$ and $x_{j}$ is determined by the gray value the degree of similarity the vector $v\left(N_{k}\right)$ and $v\left(N_{j}\right)$ of, the two the similarity of vectors is described as a weighted Euclidean distance, among them a is a Standard deviation of the Gaussian kernel function, and meet $a>0$ If a pixel structure of the neighborhood with similar structure to the gray neighborhood $x_{j}$ Then the pixel is relatively large weight, that should be a relatively large impact $x_{j}$. Weight is calculated as:

$$
\omega_{n l}\left(x_{k}, x_{j}\right)=\frac{1}{Z\left(x_{j}\right)} U\left(x_{k}, x_{j}\right)
$$

Among them $U\left(x_{k}, x_{j}\right)$ is the similarity of the exponential form, $Z\left(x_{j}\right)$ for the regularization constraints

$$
\begin{aligned}
& U\left(x_{k}, x_{j}\right)=e^{-\frac{\left\|v\left(N_{k}\right)-v\left(N_{j}\right)\right\|_{2, a}^{2}}{h^{2}}} \\
& Z\left(x_{j}\right)=\sum_{k} e^{-\frac{\left\|v\left(N_{k}\right)-v\left(N_{j}\right)\right\|_{2, a}^{2}}{h^{2}}}
\end{aligned}
$$

Parameter $\mathrm{h}$ is the control parameter, which controls the degree of exponential decline, the same, and also control the recession of the Euclidean distance weight function. For convenience of calculation, for the search similar to neighborhood structure is often limited to a program called "search window", use $\Omega_{i}$. For example, in [3] experiments, the size of neighborhood is defined as the 7 $\times 7$ square, the search window size is set to $21 \times 21$.

To determine the parameters $\lambda_{j i}$ in the formula (1), proposed called "sorting means algorithm" approach in here. This algorithm and Garnett and other peoples [4] proposed statistical method is very similar to ROAD. Suppose $x_{j}$ is the pixels we have to consider, the size of the search window $\Omega_{i}$ is $S \times S$, for each pixel in the search window, by the formula (6) to calculate the index of similarity, and then descending order of their values, then the pixelsthe balance parameter $x_{j}$ is defined as:

$$
\lambda_{j}=\frac{1}{m} \sum_{i=1}^{m} U_{i}\left(x_{k}, x_{j}\right)
$$

Among them, $U_{i}$ ranking in the search window is the U-value of $i$ first big, $m$ value is defined as $m=S-1$.

\section{EXPERIMENTAL RESULTS}

In this section, the improved FCM algorithm is applied to synthetic square image, include simulated brain images and real brain images, and results were compared with the expansion of the standard FCM algorithm. In experiments, additional brain tissue (such as the cerebral cortex, fat, etc.) has been removed before the split.

\subsection{MRI Images}

Here, we will apply the proposed algorithm in this paper in the T1 magnetic resonance brain image data. We also split the image into three categories, namely, white matter (WM), gray matter (GM) and cerebrospinal fluid (CSF), in the experiment, we do not consider the background pixels. As opposed to synthetic square image, the brain images are much more complex, so in order to 
save the image in detail, we set the parameters $h=500$, the neighborhood size $N_{j}=3 \times 3$, the search window size $\Omega_{i}=7 \times 7$.

Figure 1(a) contains a 9\% noise images of brain slices, using the standard FCM, FCMSI, IFCM, RFCM and ASFCM image segmentation algorithm, the results were as shown in Figure 1(b) to Figure 1(f). Figure 1(g) is the use of segmentation algorithms proposed in this paper, after the results. Figure 1(a) The Ground Truth as shown in Figure 1(h).

To test the proposed algorithm in this paper in different noise levels in the segmentation results, we conducted the following comparative experiments. Figures 2(a), (e) and (i) respectively, contain 3\%, 5\% and $7 \%$ of the noise of the MRI brain images, using segmentation algorithm proposed in this chapter, the results are, respectively, Figures 2(b), (f) and (g). These

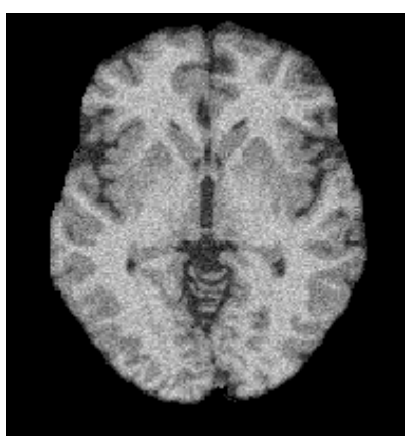

(a)

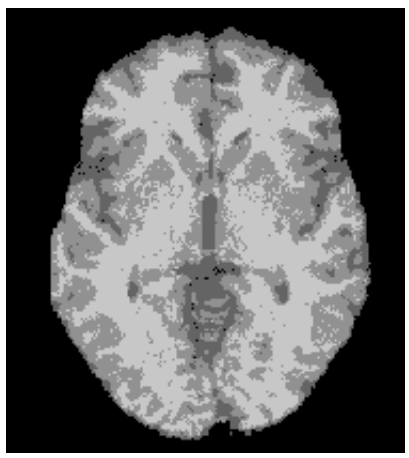

(c)

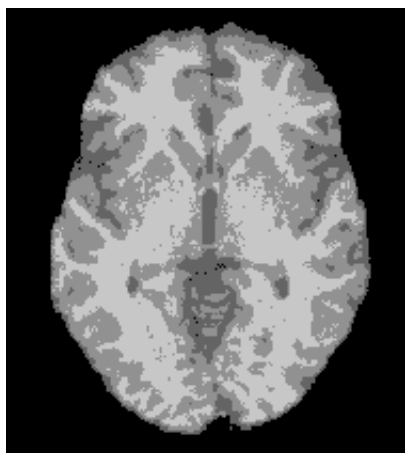

(e)

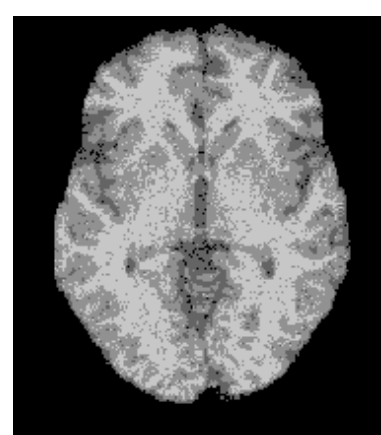

(b)

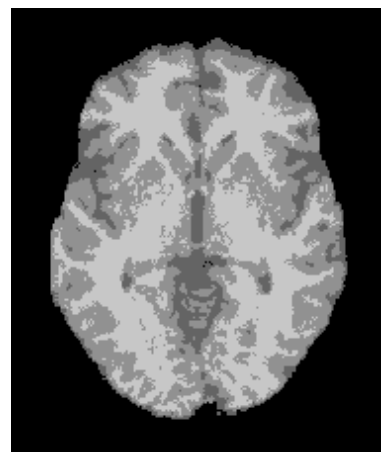

(d)

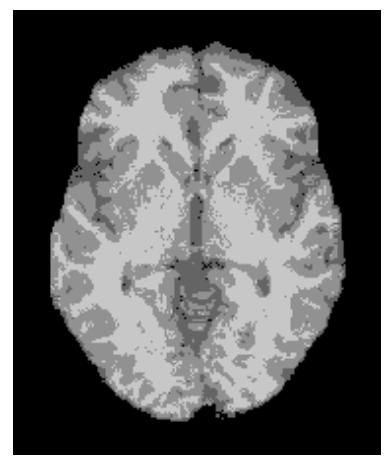

(f)

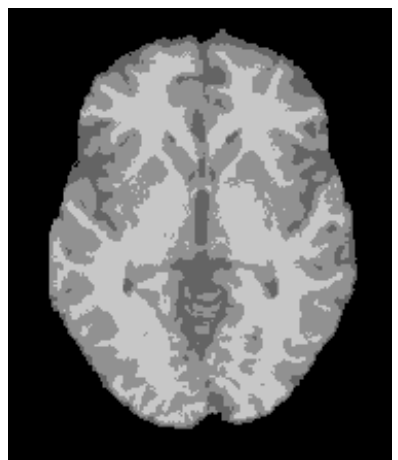

(g)

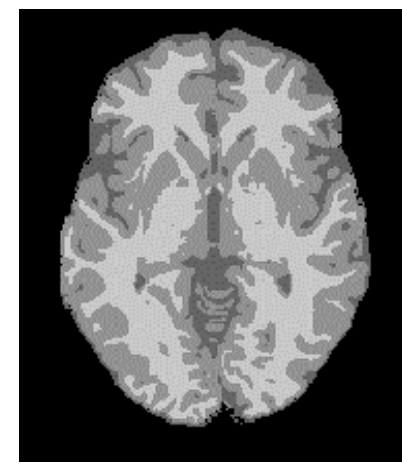

(h)

Figure 1. MRI brain images of different methods of comparing the results of Segmentation (a) brain image $(\mathrm{z}=70)$ damaged by $9 \%$ noise, (b) FCM. (c) FCMSI. (d) IFCM. (e) RFCM. (f) ASFCM. (g) The proposed method in this paper. (h) Ground Truth.

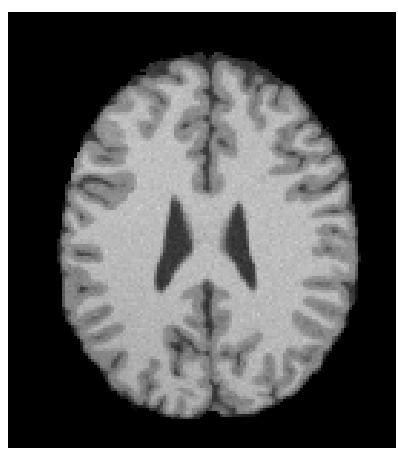

(a)

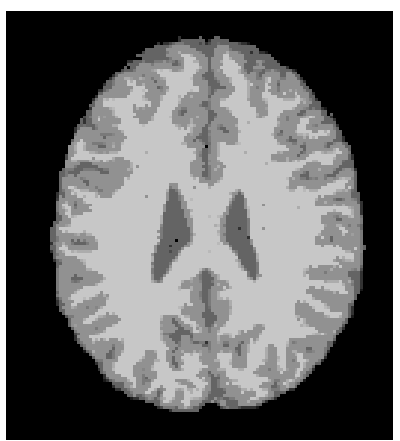

(c)

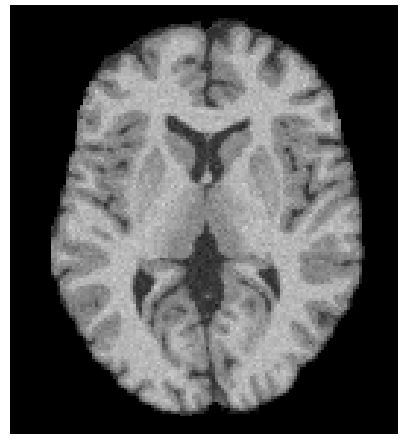

(e)

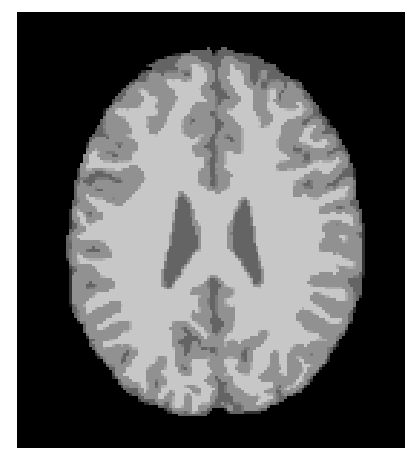

(b)

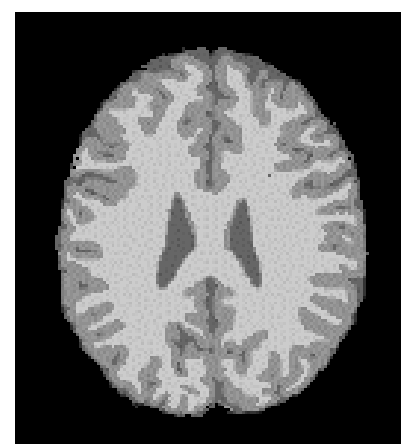

(d)

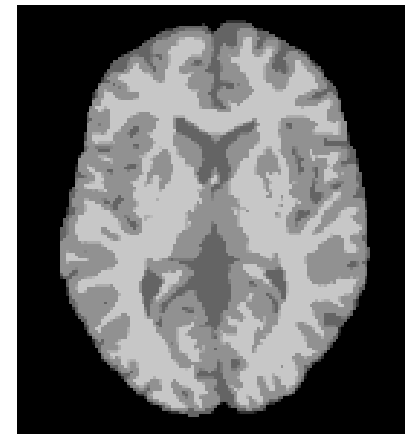

(f) 


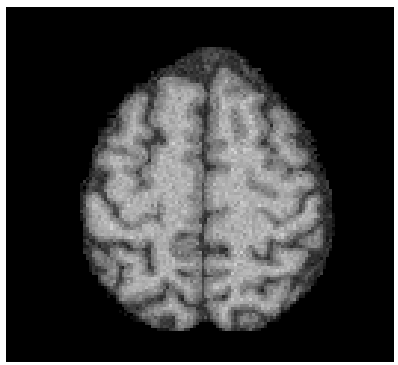

(g)

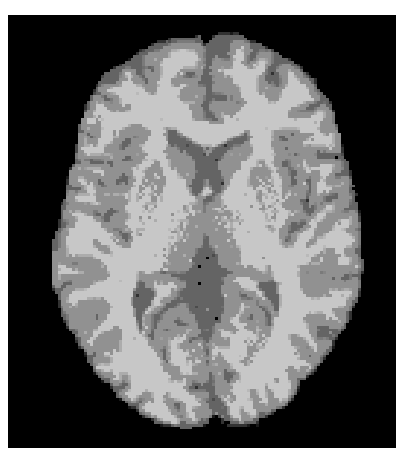

(i)

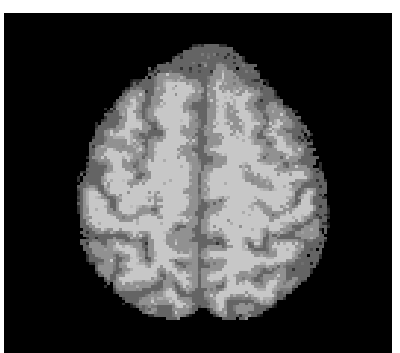

(k)

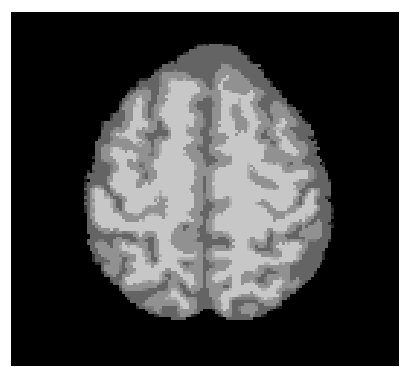

(h)

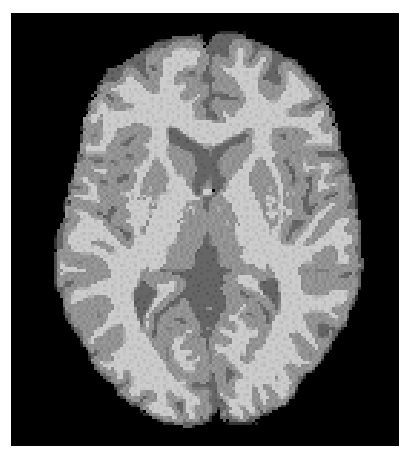

(j)

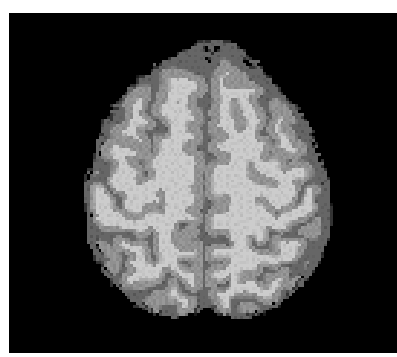

(1)
Figure 2. Segmentation results under different noise levels compared (a), (e) and (i) contain 3\%, respectively, 5\% and 7\% of the noise of the image. (b), (f) and (j) are the application of the segmentation algorithm proposed. (c), (g) and (k) are the segmentation results. (d), (h) and (l) are Ground Truth.

results with the standard FCM segmentation results and Ground Truth compare the accuracy of our method can obviously be shown.

Next, the six algorithms are applied in the different noise levels of the three-dimensional brain image segmentation; this division is carried out by the order biopsy. It can be seen, when the image does not contain noise or low noise level, all the results generated by those algorithm are the same but with the noise level enhanced, in contrast to several other algorithms, the segmentation algorithm proposed in this paper.

\section{CONCLUSION}

Because of time-consuming and subjective factors, the artificial segmentation of brain MR images results is not good repeatability. Therefore, automatic segmentation method of brain tissue is needed to complete the Automatic segmentation of MR images. But the morphology of brain tissue structure for the complex maneuver, combined with noise, partial volume effect (PVE) and image bias field (BF) existing the division of their organization with strong pixel ambiguity and uncertainty, which makes the fuzzy clustering compared to other technologies are more widely used in brain MR image segmentation. In this paper, proposed a modified fuzzy $\mathrm{C}$ means clustering algorithm. In medical images, there is often a large noise to exist. The noise can seriously affect the accuracy of the segmentation results. This method use of non-pixel neighborhood information to suppress the image of the noise, through a new distance calculation method to replace Euclidean distance measure algorithm of the traditional fuzzy $\mathrm{C}$ means to achieve the process of denoising in the during division. Through a large number of experiments and different algorithms comparison, proved the validity and correctness of the algorithm.

\section{REFERENCES}

[1] Buades, A., Coll, B. and Morel, J.M. (2005) A non-local algorithm for image denoising. IEEE Computer Society Conference on Computer Vision and Pattern Recognition, 2, 60-65.

[2] Buades, A., Coll, B. and Morel, J.M. (2004) On image denoising methods. Technical Report 2004-15, CMLA.

[3] Garnett, R., Huegerich, T., Chui, C. and He, W.J. (2005) A universal noise removal algorithm with an impulse detector. IEEE Transactions on Image Processing, 14, 1747-1754. doi:10.1109/TIP.2005.857261

[4] Johnston, B., Atkins, M.S., Mackiewich, B., et al. (1996) Segmetation of multiple selerosis lesions in intensity corrected multispectral MRI. IEEE Transactions on Medical Imaging, 15, 154-169. doi:10.1109/42.491417

[5] Suri, J.S., Singh, S. and Reden, L. (2002) Computer vision and pattern recognition techniques for $2 \mathrm{D}$ and $3 \mathrm{D}$ MR cerebral cortical segmentation (Part I): A state-ofthe-art review. Pattern Analysis \& Application, 5, 46-76. doi: $10.1007 / \mathrm{s} 100440200005$

[6] Suri, J.S., Singh, S. and Reden, L. (2002) Fusion of region and boundary/surface-based computer vision and pattern recognition techniques for $2 \mathrm{D}$ and $3 \mathrm{D}$ MR cerebral cortical segmentation (Part II): A state-of-the-art review. Pattern Analysis \& Application, 5, 77-98. doi:10.1007/s100440200006

[7] Kapur, J.N., Sahoo, P.K. and Wong, A.K.C. (1985) A new method for gray-level picture thresholding using the entropy of the histogram. Computer Vision, Graphics and Image Processing, 29, 273-285. doi:10.1016/0734-189X(85)90125-2

[8] Abutaleb, A.S. (1989) Automatic thresholding of graylevel pictures using two-dimension entropy. Computer Vision, Graphics and Image Processing, 47, 22-32.

[9] Styner, M., Brechbuhler, C. and Szckely, G. (2000) Parametric estimate of intensity inhomogeneities applied to 
MRI. IEEE Transactions on Medical Imaging, 19, 153-165. doi: $10.1109 / 42.845174$

[10] Bezdek J. (1980) A convergence theorem for the fuzzy ISODATA clustering algorithms. IEEE Transactions on
Pattern Analysis and Machine Intelligence, 2, 1-8. doi:10.1109/TPAMI.1980.4766964 